\title{
11 Wader Station Laboratory Working Routine
}

The routines and rules of the wader ringing station are very similar to those of the passerine station. Attention should be paid to recommendations resulting from different trapping methods and a slightly different set of measurements.

When at the station, species vulnerable to a long retention should be ringed first, i.e. godwits, curlews, whimbrels, greenshanks and spotted redshanks. If any passerine were brought, e.g. wagtails, they should be ringed in a second turn. After ringing, the age and, and if possible, the sex of the bird should be noted together with the ring number. This usually requires opening the wing, and so the moult score and other notes referring to the wing may be taken at the same time, e.g. features connected with the colouring of feathers and the wing length can be measured. Once the ruler is held in hand, the next measurement taken should be the tarsus + toe length. After that the callipers are produced in order to measure total head length, bill length and possibly other measurements taken with this tool (nalospi, tarsus). Finally, the bird is weighed.

In order to shorten the time of retention, the best thing is to work with a 3-4person team while ringing. One person takes birds out of the basket and clenches the ring, another takes all the measurements, a third weighs the bird and releases it, while the fourth one takes notes; alternatively, the third person takes notes while not weighing birds). When releasing waders in the daytime, they will fly away by themselves. Larger species may be carefully thrown in the air, taking care that there are no bushes or lines in their way. At night a bird taken out of the laboratory should be given time to adapt to darkness before being released. This lasts longer than in the daytime, and therefore the person weighing birds cannot release them at the same time. The bird must, under no circumstances, be allowed to leave the ringing station "on foot". If it is unable to fly but can walk and is not injured, it is better to bring it to the feeding place and release it there. After some time, most of these "immobilized" birds will fly again. 